\title{
An intervention trial to determine the response of vitamin $B 12$ biomarkers to chronic supplementation with low dose vitamin B12 after folate repletion
}

\author{
K. Pentieva ${ }^{1}$, C. Hughes ${ }^{1}$, N. Askin ${ }^{1}$, L. Hoey ${ }^{1}$, A. Molloy ${ }^{2}$, J. Scott $^{3}$ and H. McNulty ${ }^{1}$ \\ ${ }^{1}$ Northern Ireland Centre for Food and Health, University of Ulster, Coleraine, BT52 1SA, UK, ${ }^{2}$ School of Clinical \\ Medicine, Trinity College Dublin 2, Ireland and ${ }^{3}$ School of Biochemistry and Immunology, Trinity College Dublin 2, \\ Republic of Ireland
}

Mandatory folic acid (FA) food fortification has been introduced in various countries, with the primary aim of preventing neural tube defects. It has been proposed that the inclusion of vitamin B12 together with FA may provide added health benefits and alleviate the problem of 'masking' of vitamin B12 deficiency by FA in the elderly ${ }^{(1-2)}$. However, the response to supplementation with vitamin B12 at low doses has not been sufficiently investigated to date. The aim of the current study was to establish the minimum effective dose of vitamin B12 required to optimise B12 status and lower homocysteine.

A 27-week, double-blinded, placebo controlled dose-response trial was conducted. Participants ( $n$ 231), healthy younger and older adults, initially took folic acid ( $400 \mu \mathrm{g} /$ day) for 11 weeks for repletion of folate status (the main determinant of homocysteine), and were then stratified according to homocysteine concentrations and randomised to receive one of the four treatments for 16 weeks: folic acid + placebo; folic acid $+3 \mu \mathrm{g} /$ day B12; folic acid $+10 \mu \mathrm{g} /$ day B12; folic acid $+50 \mu \mathrm{g} /$ day B12. An analysis of the B12 supplements at each dose was conducted in order to confirm their actual vitamin B12 content.
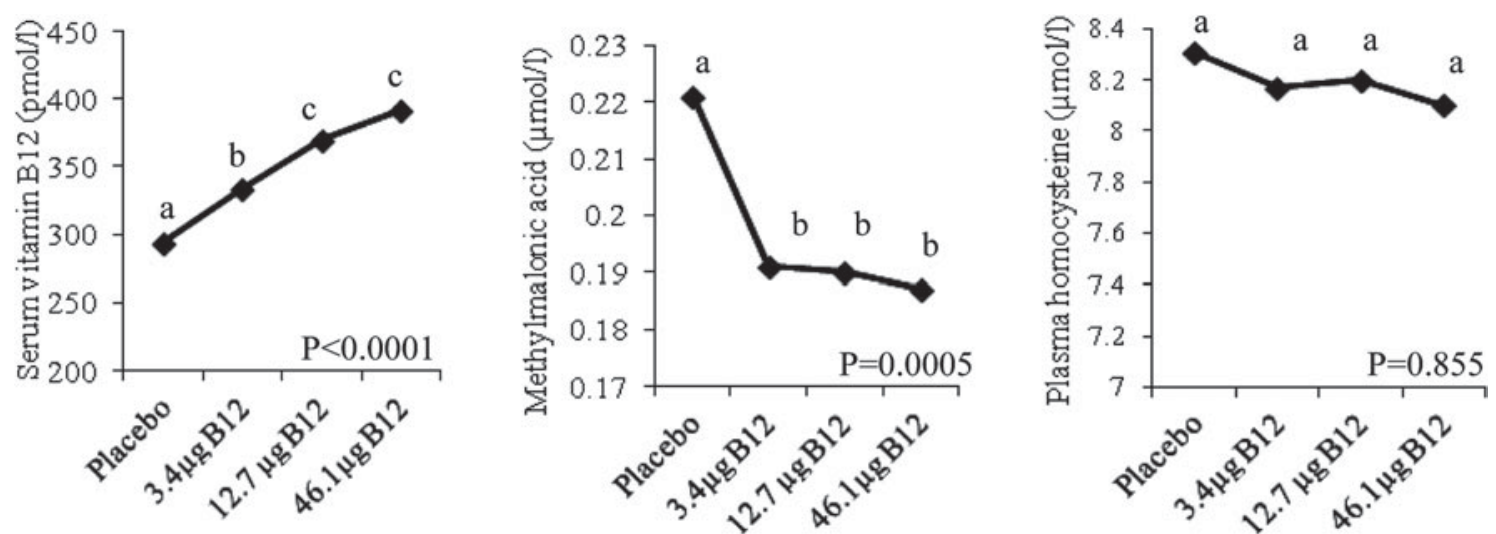

Responses of vitamin B12 biomarks to supplementation with B12 for 16 weeks. Values are geometric means. Differences between reponses were assessed by ANOVA with Tukey post hoc test.

The results showed a progressive increase in the serum vitamin B12 response to increasing doses of B12. Concentrations of methylmalonic acid (MMA), a specific functional biomarker of vitamin B12 status, were lowered by $13 \%$ and reached a plateau with a B12 dose of $3.4 \mu \mathrm{g} / \mathrm{day}$; higher doses did not result in significant further lowering. A homocysteine lowering effect of $\sim 4 \%$ was achieved in response to intervention with vitamin B12 (over and above the observed response to FA) however this was not significant at any B12 dose.

The current study demonstrates that long term intervention with low doses of vitamin B12 in healthy younger and older adults results in improved B12 status as evidenced by increased serum vitamin B12 and reduced serum MMA concentrations. The maximal response of MMA was achieved in response to supplementation with $3.4 \mu \mathrm{g} /$ day vitamin B12 in addition to the average dietary B12 intake of $4.0 \mu \mathrm{g} / \mathrm{day}$ estimated in this cohort. These findings will be of relevance to emerging dietary vitamin B 12 recommendations in that they suggest that a daily intake of around $7 \mu \mathrm{g} /$ day B12 may be considered adequate for optimising functional status of vitamin B12.

1. Czernichow S, Noisette N, Blacher J et al. (2005) Semin Vasc Med 5, 156-162.

2. Oakley GP (2002) BMJ 324, 1348-1349. 